\title{
Evidence for Beta Adrenoceptors in Proximal Tubules Isoproterenol-sensitive Adenylate Cyclase in Pars Recta of Canine Nephron
}

\author{
Naoki Murayama, Brian T. Ruggles, Susan M. Gapstur, Julie L. Werness, and Thomas P. Dousa \\ Nephrology Research Unit, Division of Nephrology and Internal Medicine, Departments of Medicine and Physiology, \\ Mayo Clinic and Foundation, Mayo Medical School, Rochester, Minnesota 55905
}

\begin{abstract}
Observations in vivo suggest that catecholamines modulate reabsorptive functions of proximal tubules by acting on $\beta$ adrenoceptors. However, $\beta$-catecholamine binding sites or $\beta$ adrenoceptor-sensitive adenylate cyclase (AdC) has not been found in segments of proximal tubules of rat, rabbit, or mouse kidney. In the present study, we investigated the responsiveness of AdC to catecholamines, [8-Arg]vasopressin (AVP), and to parathyroid hormone (PTH) in proximal convoluted tubules (PCT), proximal straight tubules (PST), and in late distal convoluted tubules (LDCT) microdissected from canine kidney. Isoproterenol (ISO) caused a marked and dose-dependent stimulation of AdC in PST (maximum: $\Delta+\mathbf{8 5 0} \%$; half maximum stimulation at $10^{-7} \mathrm{M}$ ISO), but ISO had no effect on AdC in PCT. The AdC in both PCT and PST was markedly stimulated by PTH; AVP stimulated the AdC in LDCT but not in PST or in PCT. The stimulatory effect of $10^{-5} \mathrm{M}$ ISO in PST $(\Delta+725 \%)$ was significantly greater than in LDCT $(\Delta+307 \%)$; norepinephrine and epinephrine had stimulatory effects in PST similar to ISO. The stimulation of AdC in PST by ISO was blocked by propranolol and by $\boldsymbol{\beta}_{\mathbf{2}}$-blocker ICI118551. On the other hand, $\alpha$-blocker phentolamine and $\beta_{1}$ blocker metoprolol did not abolish the stimulation of $\mathrm{AdC}$ in PST by ISO. The accumulation of CAMP in intact PCT and PST incubated in vitro was stimulated by PTH both in PST and in PCT, but ISO elevated cAMP $(\Delta+683 \%)$ only in PST. Our results show that proximal tubules of canine nephron, PST but not PCT, contain $\beta$-adrenoceptors of $\beta_{2}$ subtype coupled to AdC. These observations provide direct evidence that the effects of catecholamines, either released from renal nerve endings or arriving from blood supply, can act directly on $\beta_{2}$-adrenoceptors located in proximal tubules, and also suggest that at least some of the catecholamine effects in proximal tubules are mediated via cAMP generation.
\end{abstract}

\section{Introduction}

It is well documented that catecholamines, either released from the nerve endings juxtaposed to tubule cells or contained in renal arterial blood, modulate reabsorption of sodium and other functions of renal tubules (1-5). Recent careful autoradiographic study has shown that all portions of rat cortical tubular nephron are under some degree of neural influence

Address correspondence and reprint requests to Dr. Dousa, 921B Guggenheim Building, Mayo Clinic.

Received for publication 22 October 1984 and in revised form 15 March 1985.

J. Clin. Invest.

(c) The American Society for Clinical Investigation, Inc. 0021-9738/85/08/0474/08 \$1.00

Volume 76, August 1985, 474-481
(6). Catecholamines are potent vasoactive agents, and it is therefore difficult to discern from measurements of tubular functions in situ whether these neurotransmitters influence the function of tubular epithelium directly or rather through an indirect mechanism, e.g., by acting on renal microvasculature (7) or via the release of intrarenal mediators, such as prostaglandins or renin $(1,3)$.

Relatively few studies addressed the question of the direct effect of catecholamines on specific tubule segments in the mammalian nephron. Binding studies with use of radiolabeled ligands, mainly derivatives of adrenoceptor antagonists, showed that in rat kidney $\beta$-adrenoceptors are localized specifically in the glomeruli and in tubular segments located distal to the descending limb of Henle's loop (8). In the rabbit kidney, using $\left[{ }^{3} \mathrm{H}\right]$ prazosin binding as a probe (9), $\alpha_{1}$-adrenoceptors were identified (9) specifically in proximal convoluted tubules (PCT) ${ }^{1}$ but not in the proximal straight tubules (PST) or in the cortical collecting tubules (CCT). Microperfusion studies performed on isolated rabbit tubular segments showed that catecholamines added from peritubular side influence fluid absorption in PCT but not in PST (10). In distal tubular segments of the rabbit kidney, catecholamines, acting apparently via $\beta$-adrenoceptors, were found to decrease the potential difference across the tubular wall of CCT and cortical connecting tubules (CNT) (11), to decrease lumen-to-bath reabsorption of chloride in CCT (12) and to decrease potassium secretion in CCT (13).

It is well recognized that most $\beta$-adrenergic effects of catecholamines are elicited by binding to $\beta$-receptors coupled to adenylate cyclase (AdC) and via generating cyclic $3^{\prime}, 5^{\prime}$ AMP(cAMP) as an intracellular "second messenger" $(14,15)$. All studies to date employing segmental analysis of AdC in the rat, rabbit, and mouse nephron have shown (16) that the $\beta$-adrenoceptor-linked AdC is localized only and uniquely in segments distal to the descending limb of Henle's loop (1618), and no stimulation of AdC by catecholamines was observed in segments of the proximal tubules $(17,18)$. In distal segments, the highest sensitivity of $\mathrm{AdC}$ to $\beta$-adrenergic stimuli was found mainly in CNT and CCT (16-18).

In tissue slices of canine renal cortex incubated in vitro, $\beta$-adrenergic agents, includng isoproterenol (ISO), increased cAMP levels (19). However, these observations did not discern whether catecholamines acted on specific segments of tubules and/or on other components of the canine renal cortex.

In present studies we examined the hormonal sensitivity of AdC in specific tubular segments microdissected from the

1. Abbreviations used in this paper: AdC, adenylate cyclase; AVP, [8Arg]vasopressin; CCT, cortical collecting tubule; CNT, cortical connecting tubule; DCT, distal convoluted tubule; ISO, isoproterenol; KRB, Krebs-Ringer buffer; LDCT, late distal convoluted tubule; MIX, 1-methyl-3-isobutyl-xanthine; PCT, proximal convoluted tubule; PST, proximal straight tubule; PTH, parathyroid hormone. 
dog kidney, and found that $\mathrm{AdC}$ in proximal tubules, namely in PST, is prominently sensitive to stimulation by $\beta$-adrenergic agents. These findings provide evidence that catecholamines can act directly on proximal tubular cells and also suggest that such proximal tubular $\beta$-adrenergic effects are mediated by cAMP.

\section{Methods}

Tubular segments were microdissected from the canine kidney in a similar manner as in our other studies of nephrons from other mammalian species (20-24). Kidneys were taken from the mongrel dogs, anesthetized with pentobarbital, and immediately perfused with heparinized ice-cold collagenase medium. The tubular segments, PCT, PST, and segments of late distal cortical convoluted tubules (LDCT) were microdissected and measured as described below.

After the perfusion with heparinized collagenase medium, the cortical tissue was sliced with a razor blade and the pieces of tissue were incubated in aerated collagenase medium at $35^{\circ} \mathrm{C}$ for $50-70 \mathrm{~min}$, which was similar to our previous studies (20-22). After incubation, the slices were thoroughly rinsed in ice-cold microdissection medium and transferred to Petri dishes for microdissection. All subsequent steps were performed at $0^{\circ}-4^{\circ} \mathrm{C}$. Segments of PCT, PST, and LDCT were teased out from cortical areas of the renal tissue and identified, basically using the criteria employed previously for mammalian nephrons $(24,25)$ with some modifications. Cortical tubules of canine kidney have some features that are different from kidneys of other commonly employed experimental animals. In a morphologic survey of canine kidney, Bulger et al. (26) found that the dog proximal tubules consists of four $\left(S_{1}-S_{4}\right)$ rather than three $\left(S_{1}-S_{3}\right)$ subsegments, and that the main difference lies in the pars recta (26). The earlier portion of the proximal straight tubule $\left(\mathrm{S}_{3}\right)$ in dog is analogous to $S_{3}$ subsegment of other mammalian species, and is followed by subsegment $S_{4}$. Subsegment $\mathrm{S}_{4}$ consists of cuboidal cells with decreasing cell height and tubular diameter, and tapers towards junction with the thin descending limb of Henle's loop (26). In microdissected tubules, it is rather difficult to differentiate between $S_{3}$ and $S_{4}$. In this study, we dissected proximal straight tubules mainly from the deep cortex, and we dissected out the last tapered portion $\left(S_{4}\right)$ that dips into medulla. Therefore, in the present study, the PCT were the early convoluted portions-subsegment $S_{1}$-and the PST corresponded basically to $S_{3}$, sometimes with small initial portion of $\mathrm{S}_{4}$ (Fig. 1).

The microanatomical structure of cortical distal tubules of dog kidney was similar to the rabbit kidney as judged from appearance under the stereomicroscopic observation $(24,27,28)$. The transition between the early bright portion of the distal convoluted tubule (DCT) into the following granular portion of DCT was very clear. The granular portion of CCT resembled the granular portion of DCT. The granular portion of DCT and granular portion of CCT were not clearly

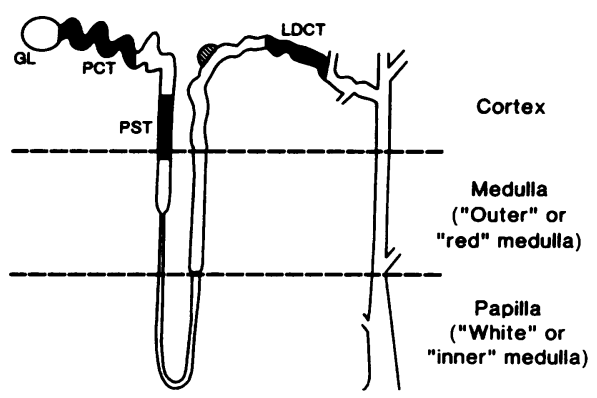

Figure 1. Schematic depiction of nephron segments from canine kidney used in the present study. Tubular regions that were dissected and analyzed are shown as black areas. GL, glomerulus. For detailed description, see Methods. separated in canine tubules. We dissected the late granular portion of DCT together with beginning part of granular portion of CCT. This segment, as shown diagramatically on Fig. 1, was operationally designated as LDCT. The LDCT (Fig. 1) approximately corresponds to the most distal parts of DCT and first portion of CNT, as described by others, for kidney of rabbit, rat, and mouse $(9,11,12,17,27,28)$.

Tubular segments were transferred onto small round glass coverslips and the total length of the tubules in the sample was determined. Samples were placed on the stage of the microscope with the drawing attachment and inspected under 100 times magnification (camera lucida). Tubules in the sample and 1-mm calibration grid were quickly drawn on white paper with a marker. The total tubular length was measured using Dietzger planimeter from the drawn pictures of samples (21). The measured samples were either immediately frozen for storage at $-80^{\circ} \mathrm{C}$ for $\mathrm{AdC}$ assay or kept at $0^{\circ}-4^{\circ} \mathrm{C}$ before incubations to determine cAMP accumulation $(21,22)$.

Before AdC assay, tubules were permeabilized by freezing and thawing in hypotonic medium $(20,24)$. The assay for AdC was identical in every detail to that described originally by Morel et al. (24) and used in our previous studies on microdissected nephron segments (20-22). AdC activity was expressed as femtomoles cAMP generated per $30 \mathrm{~min} / \mathrm{mm}$ of tubule length (20-22).

Measurement of cAMP accumulation was conducted on freshly dissected tubules using the same procedure described in every detail in our previous studies on tubules of rat or mouse kidneys (20-22). Briefly, samples of freshly dissected tubule segments were incubated in modified Krebs-Ringer buffer $(\mathrm{KRB})$ for $10 \mathrm{~min}$ at $30^{\circ} \mathrm{C}$. The incubation was stopped by placing slides on dry ice. The content of cAMP was then determined using radioimmunoassay as described in detail in previous reports (20-22). Results were expressed in femtomoles of cAMP per millimeter of total tubule length in the sample $(21,23,24)$.

As in all our previous studies (20-23), the experiments were conducted on a paired basis. The effects of agonists and antagonists were studied on tubules microdissected from the same kidney tissue and divided randomly into individual samples. Control (basal value) and experimental (hormone or drug-treated) samples were assayed and analyzed at the same time using the same reagents. Also, when

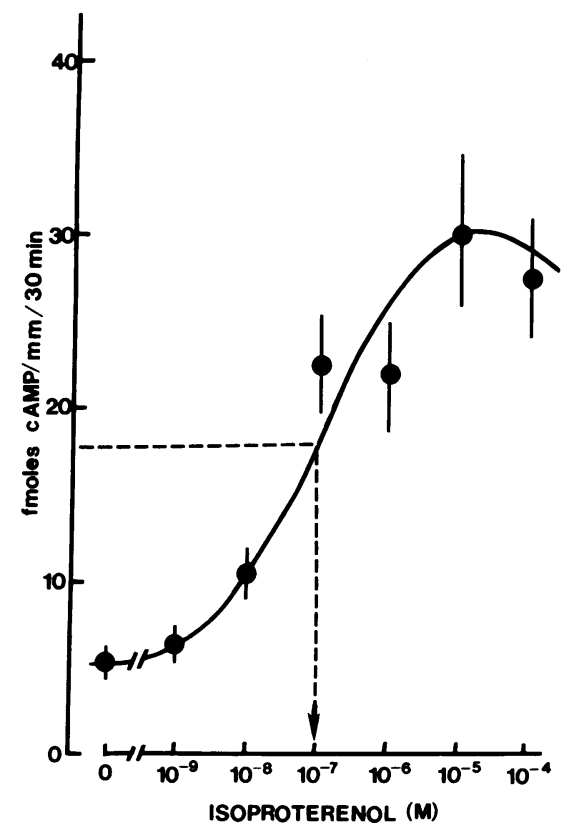

Figure 2. Dose-dependent stimulation of AdC in PST from canine kidney by ISO. Ordinate: specific activity of AdC; abscissa: molar concentration of ISO. Each point denotes mean \pm SEM of 9-12 samples. Interrupted line indicates concentration of ISO that elicited onehalf maximum increase in AdC activity over the basal value. 


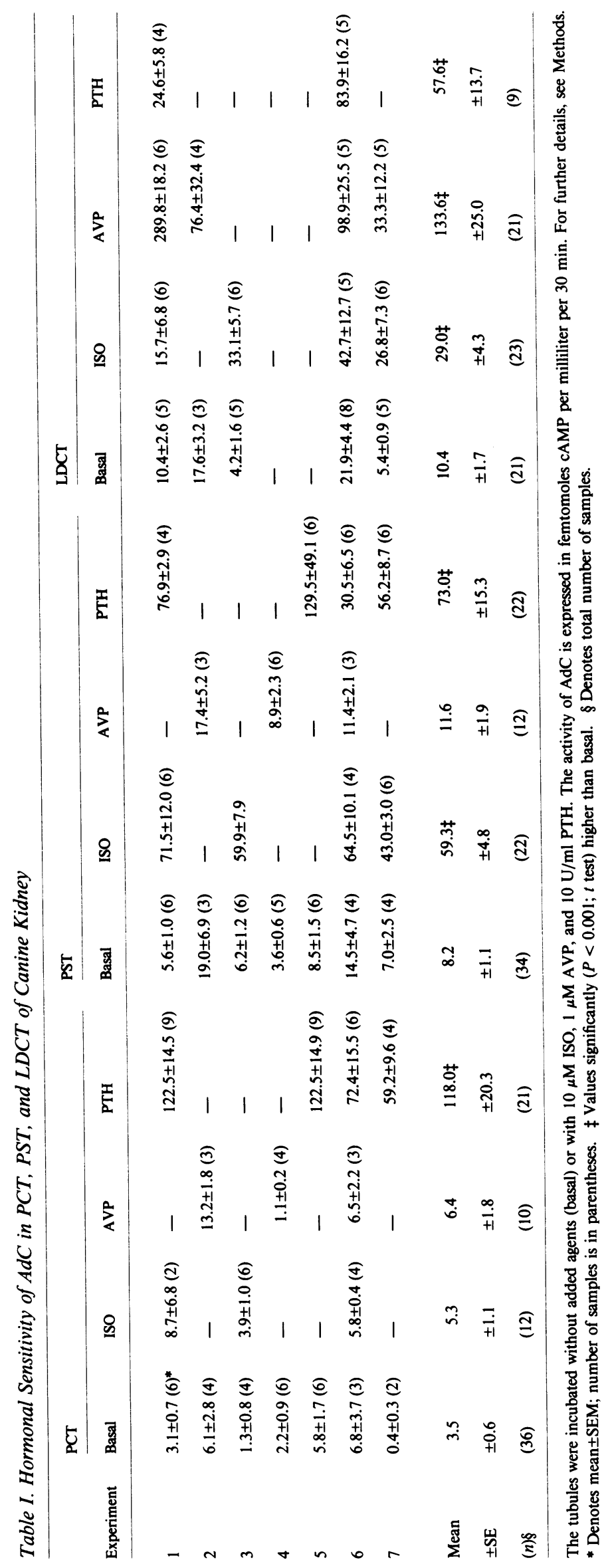



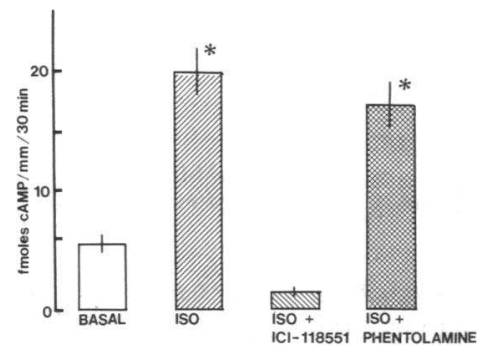

Figure 3. Effect of $\beta_{2}$-antagonist (ICI-1 18551) and $\alpha$ antagonist (phentolamine) on the ISO-stimulated activity of AdC in PST from canine kidney. Each bar denotes mean \pm SEM of 914 samples pooled from two kidneys. Concentration of ISO was $1 \mu \mathrm{M}$, and concentrations of antagonists were $10 \mu \mathrm{M}$. *, Value significantly $(\boldsymbol{P}$ $<0.001 ; t$ test) higher than the basal activity. Activity of AdC with ISO alone (घ) and the AdC activity with ISO plus phentolamine (घ) were not significantly different from each other.

compared, the cortical tubular segments (PCT, PST, and LDCT) were dissected from the same kidney and assayed simultaneously. Results were evaluated by $t$ test; values of $\boldsymbol{P}>0.05$ were considered nonsignificant (NS).

Solution and materials. The microdissection medium was a modified Hanks' balanced salt solution described in detail in our previous studies (24-26) and contained in final concentration $137 \mathrm{ml} \mathrm{NaCl}, 5$ $\mathrm{mM} \mathrm{KCl}, 0.8 \mathrm{mM} \mathrm{MgSO}_{4}, 0.33 \mathrm{mM} \mathrm{NaH} \mathrm{PO}_{4}, 1 \mathrm{mM} \mathrm{MgCl}, 10$ $\mathrm{mM}$ Tris $\mathrm{HCl}$, and $0.25 \mathrm{mM} \mathrm{CaCl}, \mathrm{pH}$ 7.4. The composition of collagenase medium was identical to that of microdissection medium, except that the $\mathrm{CaCl}_{2}$ concentration was $1 \mathrm{mM}$ and also contained bovine serum albumin $(0.1 \% \mathrm{wt} / \mathrm{vol})$, hyaluronidase $(0.1 \% \mathrm{wt} / \mathrm{vol})$, and collagenase $(0.15 \% \mathrm{wt} / \mathrm{vol})$. The hypoosmotic medium was also described previously (23-25). Modified KRB contained $140 \mathrm{mM} \mathrm{NaCl}, 5 \mathrm{mM}$ $\mathrm{KCl}, 1.2 \mathrm{mM} \mathrm{MgSO}, 0.8 \mathrm{mM} \mathrm{CaCl}, 10 \mathrm{mM}$ sodium acetate, 10 $\mathrm{mM}$ glucose, $20 \mathrm{mM}$ Tris, and $2 \mathrm{mM} \mathrm{NaH} \mathrm{PO}_{4}$ (pH 7.4). In all incubations to determine cAMP accumulation, KRB also contained $0.5 \mathrm{mM}$ 1-methyl-3-isobutyl-xanthine (MIX).

In preliminary experiments, some assays were conducted in the presence of $5 \times 10^{-6} \mathrm{M}$ ascorbic acid, an antioxidant (18). Results were not different from assays without such addition. The stock solutions of adrenergic agonists and blockers were always prepared fresh, before the assays, to prevent possible inactivation. Other hormones, [8-Arg] vasopressin (AVP) and parathyroid hormone (PTH), were from aliquots of stock solutions, prepared and stored in similar way as described previously (21-23).

Materials. Bovine serum albumin, collagenase (type I, $150 \mathrm{U} / \mathrm{mg}$ ), hyaluronidase (types 1-5, 500 National Formulary U/mg), cAMP, and other biochemicals were purchased from Sigma Chemical Co., St. Louis, MO. Sodium heparin (Panheparin, 1,000 U.S. Pharmacopeia $\mathrm{U} / \mathrm{ml}$ ) was purchased from Abbot Laboratories Chemical Div., Chicago, IL. $\left[\alpha^{-32} \mathrm{P}\right]$ ATP $(25 \mathrm{Ci} / \mathrm{mM})$ and $\left[{ }^{3} \mathrm{H}\right] \mathrm{cAMP}(30-50 \mathrm{Ci} / \mathrm{mM})$ were purchased from ICN Nutritional Biochemicals, Cleveland, $\mathrm{OH}$. Synthetic AVP (385 U/mg) was purchased from Calbiochem Behring Corp.,
American Hoechst, San Diego, CA. The radioimmunoassay kits for measurement of cAMP were purchased from New England Nuclear, Boston, MA. Synthetic bovine $\mathrm{NH}_{2}$-terminal (1-34) parathyroid hormone $(5,200 \mathrm{U} / \mathrm{mg})$ was purchased from Beckman Instruments, Inc., Palo Alto, CA). D,L-ISO, L-norepinephrine, L-epinephrine and D,Lpropranolol $\mathrm{HCl}$ were purchased from Sigma Chemical Co. Metoprolol ( $\alpha_{1}$-blocking agent) and phentolamine ( $\alpha$-blocking agent) were products of CIBA-Geigy Co., Summit, NJ. The specific $\beta_{2}$-blocking agent ICI118551 (erythro-DL-1 [7-methylindan-4-yloxy]-3-isopropyl-aminobutan2ol) was a product of Imperial Chemical Industries, Cheshire, England. These compounds were generous gifts from Dr. Paul Vanhoutte, Department of Physiology, Mayo Clinic and Foundation. All other compounds and reagents, all of the highest purity grades available, were purchased from standard suppliers.

\section{Results}

As a first step, we examined the response of AdC to ISO and some other hormones in PCT and PST. Since the distal tubular segments (CNT, CCT, and DCT) were reported to contain ISO-sensitive AdC in previously studied mammalian species $(16-18,27,28)$, the responsiveness of AdC to ISO was also measured in LDCT. The AdC was stimulated by PTH both in PCT ( $>30$ times) and in PST ( 9 times), but no stimulatory response to AVP was detected (Table I); PCT and PST differed strikingly in their response to ISO (Table I). While, in PST, $10 \mu \mathrm{M}$ ISO caused a prominent (seven times) increase in AdC activity, it had no stimulatory effect on AdC in PCT from the same kidney (Table I). Both ISO and AVP stimulated AdC in LDCT (Table I). The extent of the increase in AdC activity over the basal (expressed in femtomoles cAMP per millimeter per $30 \mathrm{~min})$ in response to $10 \mu \mathrm{M}$ ISO in PST $(\Delta+51.4 \pm 6.1$, mean \pm SEM; $n=4)$ was significantly $(P<0.01, t$ test $)$ greater than the net stimulation of $\operatorname{AdC}(\Delta+19.0 \pm 4.9$, mean \pm SEM; $n=4$ ) in LDCT of the same kidney; the basal activity of AdC was not different between PST and LDCT (Table I). The stimulation of AdC by ISO in canine PST was dose dependent (Fig. 2). The half maximum stimulation was achieved at an ISO concentration of $\sim 0.1 \mu \mathrm{M}$, and the maximum plateau was reached at $\sim 10 \mu \mathrm{M}$ ISO (Fig. 2).

The AdC in PST of canine kidney was stimulated not only by synthetic $\beta$-agonist ISO but also by norepinephrine (Table II) and by epinephrine (Table III $A$ ). The nonselective $\beta$ blocker propranolol completely prevented the stimulation of AdC in PST by ISO and by norepinephrine (Table II), but did not diminish AdC activity stimulated by PTH (Table III $B$ ). On the other hand, the $\alpha$-blocker phentolamine did not inhibit

Table II. Effect of $\beta$-Adrenergic Agonists and Antagonists on AdC Activity in PST of Canine Kidney

\begin{tabular}{|c|c|c|c|c|c|c|c|}
\hline Experiment & Basal & $1 \mu \mathrm{M}$ ISO & $\begin{array}{l}1 \mu \mathrm{M} \text { ISO } \\
+10 \mu \mathrm{M} \text { propranolol }\end{array}$ & $\begin{array}{l}1 \mu \mathrm{M} \text { ISO } \\
+10 \mu \mathrm{M} \text { metoprolol }\end{array}$ & $\begin{array}{l}1 \mu \mathrm{M} \text { ISO } \\
+10 \mu \mathrm{M} \text { ICI-118551 }\end{array}$ & $1 \mu \mathrm{M}$ norepinephrine & $\begin{array}{l}1 \mu \mathrm{M} \text { norepinephrine } \\
+10 \mu \mathrm{M} \text { propranolol }\end{array}$ \\
\hline 1 & $2.6 \pm 0.2(6)^{*}$ & $36.8 \pm 4.3(6)$ & - & - & - & $34.8 \pm 2.0(5)$ & - \\
\hline 2 & $4.0 \pm 1.0(4)$ & $34.4 \pm 7.4(4)$ & $2.2 \pm 1.3(6)$ & $33.6 \pm 3.2(6)$ & $3.1 \pm 0.5(6)$ & $20.5 \pm 2.5$ & $6.5 \pm 1.9(6)$ \\
\hline 3 & $4.5 \pm 0.6(6)$ & $26.5 \pm 2.5(8)$ & $2.7 \pm 0.9(5)$ & $21.3 \pm 1.7(5)$ & $1.3 \pm 0.3(5)$ & $16.7 \pm 5.7(8)$ & $1.1 \pm 0.3(4)$ \\
\hline Mean & 3.6 & $31.0 \ddagger$ & 2.4 & $28.0 \ddagger$ & 2.3 & $23.0 \ddagger \S$ & 4.3 \\
\hline \pm SEM & \pm 0.4 & \pm 2.4 & \pm 0.8 & $\pm 2.7^{\circ}$ & \pm 0.4 & \pm 2.9 & \pm 1.4 \\
\hline$n^{\prime \prime}$ & (16) & (18) & (11) & (11) & (11) & (18) & $(10)$ \\
\hline
\end{tabular}

ISO and norepinephrine were used as agonists. AdC activity (femtomoles cAMP per millimeter per 30 min). * Denotes mean \pm SEM; number of samples in brackets. $¥$ Significantly higher than basal value $(P<0.001 ; t$ test). $\S$ Significantly lower than the value with $1 \mu \mathrm{M}$ ISO alone $(P$ $<0.05 ; t$ test). " Total number of samples. 
Table III. Effect of Epinephrine (A) and Propranolol (B) on AdC Activity in PST of Canine Kidney

\begin{tabular}{|c|c|c|c|c|c|c|c|}
\hline \multicolumn{3}{|c|}{ A. Effect of epinephrine of AdC in PST of canine kidney } & \multicolumn{5}{|c|}{ B. Effect of propranolol on AdC in PST of canine kidney stimulated by PTH or by ISO } \\
\hline Experiment & Basal & $10 \mu \mathrm{M}$ epinephrine & Experiment & $10 \mathrm{U} / \mathrm{ml}$ PTH & $\begin{array}{l}10 \mathrm{U} / \mathrm{ml} \text { PTH } \\
+0.1 \mathrm{mM} \text { propranolol }\end{array}$ & $10 \mu \mathrm{M}$ ISO & $\begin{array}{l}10 \mu \mathrm{M} \text { ISO } \\
+0.1 \mathrm{mM} \text { propranolol }\end{array}$ \\
\hline 1 & $6.8 \pm 1.0(6)^{*}$ & $11.1 \pm 1.4(6)$ & 1 & $33.7 \pm 0.8(6)^{*}$ & $34.5 \pm 3.7(6)$ & $14.5 \pm 0.9(6)$ & $3.9 \pm 0.6(6)$ \\
\hline 2 & $6.3 \pm 0.6(6)$ & $11.0 \pm 2.7(5)$ & 2 & $23.8 \pm 2.0(6)$ & $33.5 \pm 5.7(6)$ & $14.7 \pm 2.2(6)$ & $2.1 \pm 0.5(6)$ \\
\hline 3 & $4.6 \pm 0.7(6)$ & $10.8 \pm 1.4(6)$ & & & & & \\
\hline Mean & 5.9 & $11.0 \ddagger$ & Mean & 28.7 & 34.1 & 14.6 & $3.0 \|$ \\
\hline \pm SEM & \pm 0.5 & \pm 1.0 & \pm SEM & \pm 1.8 & \pm 3.3 & \pm 1.5 & \pm 0.5 \\
\hline$n \S$ & (18) & (17) & $n \S$ & (12) & (12) & (12) & (12) \\
\hline
\end{tabular}

AdC activity is expressed in femtomoles cAMP per millimeter per $30 \mathrm{~min} .{ }^{*}$ Denotes mean $\pm \mathrm{SEM}$; number of samples in brackets. $¥$ Signifcantly higher than basal value $(P<0.001 ; t$ test $)$. $\S$ Total number of samples. "Significantly lower than value with ISO alone $(P<0.001, t$ test).

effect of ISO (Fig. 3). The $\beta_{1}$-antagonist metoprolol did not prevent the stimulation of AdC by ISO in PST (Table II), but the specific $\beta_{2}$-antagonist ICI-118551 caused a complete inhibition (Table II).

To ascertain that the stimulatory effect of ISO on AdC in canine PST is not due to some methodological features of our assay systems, we compared effects of ISO and other hormones on AdC in PST, PCT, and LDCT from dog, rat, and rabbit kidneys microdissected and assayed simultaneously (Table IV). As outlined in Table III, the ISO stimulated AdC only in PST from canine nephron, and not in PST from rabbit and rat kidneys. On the other hand, ISO increased the activity of AdC in LDCT from all the three tested species (Table IV).

In further experiments, we examined whether ISO influences the accumulation of CAMP in the intact segments of PCT and PST microdissected from dog kidney in the similar way as it stimulates the AdC. Incubations were conducted in the presence of $0.5 \mathrm{mM}$ MIX to optimize cAMP accumulation. As with AdC assayed in permeabilized tubules, the incubation of intact PCT and PST with PTH elicited a marked elevation of cAMP content (Fig. 4). Incubation with $1 \mu \mathrm{M}$ ISO was without effect on cAMP levels in PCT, but elicited a marked increase ( $\Delta$ $+683 \%$ ) of cAMP levels in PST; AVP did not influence the cAMP accumulation in PST (Fig. 4). This stimulatory effect of ISO was blocked by propranolol and by the $\beta_{2}$-blocker ICI118551 (Table V). On the other hand, propranolol did not influence the stimulatory effect of PTH on cAMP accumulation (Table V).

\section{Discussion}

Considering the fundamental role of renal nerves and circulating catecholamines in modulation of renal function (1-5), as well as various renal effects of now widely used $\beta$-blocking agents (29), the question whether the function of proximal tubular epithelium is modulated directly via $\beta$-adrenoceptors is of major interest and potential importance.

Many effects of catecholamines are elicited at cellular level by binding on $\beta$-adrenoceptors coupled to $\operatorname{AdC}(15,16)$; our present results document that proximal tubules of canine kidney are endowed with such a $\beta$-adrenoceptor-linked AdC

Table IV. Differential Response of AdC

\begin{tabular}{|c|c|c|c|c|c|c|c|c|c|}
\hline & \multicolumn{3}{|c|}{ A. Canine nephron } & \multicolumn{3}{|c|}{ B. Rat nephron } & \multicolumn{3}{|c|}{ C. Rabbit nephron } \\
\hline & PCT & PST & LDCT & PCT & PST & LDCT & PCT & PST & LDCT \\
\hline Basal & $\begin{array}{l}0.4 \pm 0.3^{*} \\
n=2\end{array}$ & $\begin{array}{l}7.0 \pm 2.5 \\
n=4\end{array}$ & $\begin{array}{l}5.4 \pm 0.9 \\
n=5\end{array}$ & $\begin{array}{c}5.6 \pm 0.9 \\
n=4\end{array}$ & $\begin{array}{l}0.5 \pm 0.1 \\
n=3\end{array}$ & 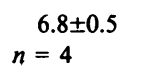 & 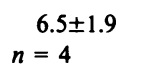 & $\begin{array}{l}1.4 \pm 0.6 \\
n=4\end{array}$ & $\begin{array}{l}25.5 \pm 1.8 \\
n=4\end{array}$ \\
\hline $1 \mu \mathrm{M}$ ISO & $\begin{array}{l}1.5 \pm 0.5 \\
n=3\end{array}$ & $\begin{array}{l}30.6 \pm 3.2 \ddagger \\
n=6\end{array}$ & $\begin{array}{l}13.2 \pm 2.4 \ddagger \\
n=5\end{array}$ & $\begin{array}{l}4.7 \pm 1.3 \\
n=3\end{array}$ & $\begin{array}{l}2.0 \pm 1.0 \\
n=3\end{array}$ & $\begin{array}{l}74.8 \pm 12.4 \ddagger \\
n=4\end{array}$ & $\begin{array}{l}3.9 \pm 0.5 \\
n=3\end{array}$ & $\begin{array}{l}2.3 \pm 0.9 \\
n=4\end{array}$ & $\begin{array}{l}63.3 \pm 13.6 \\
n=4\end{array}$ \\
\hline $\begin{array}{l}\text { PTH } \\
\quad(10 \mathrm{U} / \mathrm{ml})\end{array}$ & $\begin{array}{l}59.2 \pm 9.6 \ddagger \\
n=4\end{array}$ & $\begin{array}{l}56.2 \pm 8.7 \ddagger \\
n=6\end{array}$ & - & $\begin{array}{l}67.0 \pm 11.3 \ddagger \\
n=4\end{array}$ & $\begin{array}{l}3.0 \pm 0.7 \ddagger \\
n=4\end{array}$ & - & $\begin{array}{l}320.7 \pm 23.9 \ddagger \\
n=4\end{array}$ & $\begin{array}{l}63.8 \pm 22.1 \ddagger \\
n=4\end{array}$ & - \\
\hline $1 \mu \mathrm{M}$ AVP & - & - & $\begin{array}{l}33.3 \pm 12.2 \ddagger \\
n=5\end{array}$ & - & - & $\begin{array}{l}109.9 \pm 24.4 \ddagger \\
n=2\end{array}$ & - & - & $\begin{array}{l}65.1 \pm 24.4 \ddagger \\
n=4\end{array}$ \\
\hline
\end{tabular}

Different responses of AdC to $1 \mu \mathrm{M}$ ISO, $10 \mathrm{U} / \mathrm{ml}$ PTH, and $1 \mu \mathrm{M}$ AVP in tubular segments from dog (A), rat (B), and rabbit (C) kidney. PCT, PST, and LDCT were microdissected from the same kidney. Tubules from dog, rat, and rabbit were assayed simultaneously using the same hormones. The AdC activity is expressed in femtomoles cAMP per millimeter per 30 min. For further details, see Methods. * Mean $\pm S E M ; n$, number of samples. $¥$ Significant values $(P \leq 0.05$ or higher degree of significance), $t$ test different from corresponding basal value. 


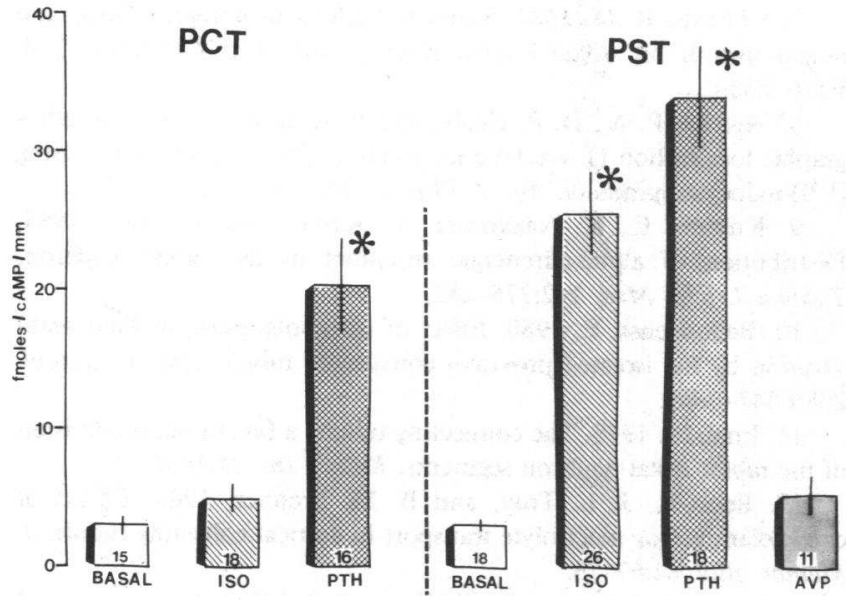

Figure 4. The content of CAMP in intact proximal tubules from canine kidney incubated with $1 \mu \mathrm{M}$ ISO, with $10 \mathrm{U} / \mathrm{ml}$ of PTH, or with $1 \mu \mathrm{M}$ AVP. (Left) PCT. (Right) PST. Each column denotes mean $\pm \mathrm{SEM}$, number of samples is indicated at the bottom of bars. Each column represents mean of samples pooled from the three independent experiments. $*$, Values significantly different $(P<0.001$; $t$ test) from corresponding basal value.

system. As in similar studies of $\beta$-adrenergic effects in specific nephron segments $(16,18)$, we employed ISO as a synthetic prototype of $\beta$-adrenergic agonist. Unlike in other species of laboratory animals, ISO stimulated AdC in PST, a subsegment of proximal tubules (Tables I and II, Fig. 2). Likewise, natural catecholamines norepinephrine (Table II) and epinephrine (Table III $A$ ) had similar stimulatory effects. It is noteworthy that in the dog kidney the extent of AdC stimulation by ISO is even greater in PST than in LDCT, a major site of ISOsensitive AdC in nephron of rat, mouse, and rabbit (16-18, 27; Table IV). It seems most unlikely that the observed interspecies difference in the sensitivity of proximal tubules to $\beta$-adrenergic stimuli might be accounted for by technical factors, since the same differential response of AdC in PST and LDCT to ISO was found on using identical experimental setting (Table IV). Otherwise, the hormonal responsiveness of

Table V. Action of $\beta$-Blocking Agents on ISO-elicited cAMP Accumulation in Intact PST

\begin{tabular}{lrr}
\hline Condition & & \\
\hline & $n$ & fmol cAMP/mm \\
Basal & $(12)$ & $3.7 \pm 0.5^{*}$ \\
$1 \mu \mathrm{M}$ ISO & $(20)$ & $28.9 \pm 3.2 \ddagger$ \\
$1 \mu \mathrm{M}$ ISO $+100 \mu \mathrm{M}$ & & \\
$\quad$ propranolol & $(13)$ & $6.0 \pm 1.3 \S$ \\
$1 \mu \mathrm{M}$ ISO $+100 \mu \mathrm{M} \mathrm{ICI}-118551$ & $(5)$ & $5.4 \pm 1.7 \S$ \\
PTH $(10 \mathrm{U} / \mathrm{ml})$ & $(6)$ & $32.5 \pm 4.9 \ddagger$ \\
PTH $(10 \mathrm{U} / \mathrm{ml})+100 \mu \mathrm{M}$ & & \\
$\quad$ propranolol & $(6)$ & $42.6 \pm 8.0 \ddagger$ \\
\hline
\end{tabular}

For details, see Methods.

* Mean \pm SEM of $n$ samples (total from 1 to 2 preparations).

¥ Significantly higher than basal value $(P<0.001 ; t$ test $)$.

$\S$ Significantly lower than value with ISO alone $(P<0.005 ; t$ test).
AdC in proximal tubules of canine nephron is similar to kidneys of other studied mammalian species, in that both PCT and PST are stimulated by PTH (Tables I and IV) but not by AVP (16-18, 23, 28, 30). Likewise, as in other species, the canine LDCT that corresponds to the most distal portion of DCT and early portion of CNT (see Methods and Fig. 1) contained the AdC sensitive to stimulation by AVP (Table I).

The stimulation of AdC in PST by ISO is a dose-dependent phenomenon (Fig. 2); in terms of the threshold concentration for stimulation and the apparent affinity, the PST resembles other mammalian cell types $(14,15,18,31)$. It may be expected that the $\beta$-adrenoreceptor-linked AdC in PST can be stimulated not only by neurotransmitters released locally from juxtaposed renal nerve endings $(1-4,6)$, but also by the circulating catecholamines. Even under resting conditions, the nanomolar concentrations of norepinephrine and epinephrine in renal arterial supply of the canine kidney (5) is around the threshold stimulatory range for ISO (Fig. 2). Even if affinity of $\beta$-receptor associated with AdC would be lesser for norepinephrine and epinephrine than for ISO (Fig. 2), circulating catecholamines, markedly elevated under pathologic conditions $(32,33)$, are likely to elicit stimulation of the AdC in PST.

Stimulation of AdC by ISO in PST is blocked by $\beta$ antagonist propranolol (Tables II and III $B$ ) but not by $\alpha$ antagonist phentolamine (Fig. 3), and this affirms that the receptors in PST are of $\beta$-types. In this respect, AdC in canine PST resembles the analogous system in CNT and CCT of rabbit nephron (18). Further indication that stimulation by ISO is specific for $\beta$-receptor is a finding that propranolol inhibits effects of ISO, but not of PTH, in the same preparation of PST (Table II $B$ ). Results of experiments with selective $\beta$ adrenergic antagonists, the $\beta_{2}$-blocker ICI-118551 (34) and $\beta_{1^{-}}$ blocker metoprolol (Fig. 3, Tables II and V), indicate that the $\beta$-adrenoreceptors linked with AdC in canine PST are of $\beta_{2}$ subtype $(14,34)$.

The hormonal modulatory effects on cAMP accumulation in the intact cells are sometimes less readily detected than the stimulation of AdC assayed in tubules permeabilized by freezing in hypotonic medium $(22,23)$. Therefore, we examined the response of canine proximal tubules to ISO and other agents also in terms of cAMP accumulation (Fig. 4). Pattern of the responses to agonists and antagonists in intact PST is virtually identical to responses of AdC measured in permeabilized (20, 24) tubules. The stimulation of AdC by ISO in the PST, but not in PCT, is reflected by a marked ISO-elicited increase of cAMP content in PST (Fig. 4, Table V). As with AdC, the stimulatory effect of ISO, but not that of PTH, on CAMP accumulation in PST was blocked by $\beta$-antagonists (Table V).

To our knowledge, this study provides first evidence that the proximal tubules of mammalian nephron contain AdC coupled to $\beta$-adrenoceptors. Our findings thus document that epithelial cells of pars recta of proximal tubules-PST-can respond to catecholamine stimulation directly, and it also suggests that the cellular response is at least in part mediated by increase in cAMP. Only direct studies on isolated canine PST and PCT will reveal which tubular function(s), transport, or metabolic are regulated by catecholamines via stimulation of $\beta_{2}$-adrenoceptor-linked cAMP system. Concerning renal effects of $\beta$-blocking agents (29), it may be useful to know that only nonselective or $\beta_{2}$-specific antagonists can influence the adrenergic receptors in cells of PST.

Finally, our results (Table IV) as well as the comparison 
with findings by others (16-18) underscore marked differences between species of mammals in terms of neuro-hormonal regulation of nephron function (35), and emphasize the caution with which experimental results on various animal models can be extrapolated to human pathophysiology. In this context it is not without interest that Bulger, Cronin, and Dobyan (26) found that the canine nephron resembles more closely the human nephron than the nephron of rat, which is perhaps the most widely used animal in experimental nephrology (30). In particular, these authors note that PST, unlike PCT, differs markedly in ultrastructure between kidney of dog and rat (26). To extend this point, in our recent study, we observed that the responsiveness of AdC to AVP and to forskolin in the distal tubular segments is virtually identical in canine and human kidney (36), but differs from rat, rabbit, and mouse $(16,21,22,28,37)$. The comparisons recounted above $(26$, 36) raise the possibility that the canine kidney may be in many ways a closer model for studies of human renal pathophysiology than kidneys of other frequently used laboratory animals.

In conclusion, we document that proximal tubules of canine kidney, namely PST, are endowed with $\beta_{2}$-adrenoceptors coupled to the AdC-cAMP system and that the sensitivity of this tubular subsegment to catecholamines is similar or even higher than in LDCT. Intrarenal or circulating catecholamines can act directly on epithelial cells of proximal tubules and such direct effects are likely mediated by cAMP. The relative contribution of this newly found $\beta_{2}$-adrenoceptor-mediated action to the overall modulation of kidney functions by the adrenergic system remains to be elucidated.

\section{Acknowledgments}

Ms. Jacquelyn Trefz and Mrs. Carolyn Blankenship provided secretarial assistance.

This study was supported by National Institutes of Health grant AM-16105, by grant-in-aid from The National Kidney Foundation of the Upper Midwest, and by the Mayo Foundation. Dr. Naoki Murayama is recipient of postdoctoral research fellowship from the American Heart Association, Minnesota Affiliate.

\section{References}

1. DiBona, G. F. 1982. The functions of the renal nerves. In Reviews of Physiology, Biochemistry and Pharmacology. R. H. Adrian, E. Helmreich, R. Jung, R. J. Linden, J. Pliper, U. Trendelenburg, and W. Vogt, editors. Springer-Verlag, Berlin-Heidelberg-New York. 75181.

2. Kim, J. K., S. L. Linas, and R. W. Schrier. 1980. Catecholamines and sodium transport in the kidney. Pharmacol. Rev. 31:169-178.

3. DiBona, G. F. 1977. Neurogenic regulation of renal tubular sodium reabsorption. Am. J. Physiol. 233:F73-F81.

4. DiBona, G. F. 1978. Neural control of renal tubular sodium reabsorption in the dog. Fed. Proc. 37:1214-1217.

5. Katholi, R. E., S. Oparil, F. Urthaler, and T. N. James. 1979. Mechanism of postarrhythmic renal vasoconstriction in the anesthetized dog. J. Clin. Invest. 64:17-31.

6. Barajas, L., K. Powers, and P. Wang. 1984. Innervation of the renal cortical tubules: a quantitative study. Am. J. Physiol. 247:F30F60.
7. Edwards, R. M. 1983. Segmental effects of norepinephrine and angiotensin II on isolated renal microvessels. Am. J. Physiol. 244: F526-F534.

8. Munzel, P. A., D. P. Healy, and P. A. Insel. 1984. Autoradiographic localization of $\beta$-adrenergic receptors in rat kidney slices using [25I]-iodocyanopindolol. Am. J. Physiol. 246:F240-F245.

9. Kusano, E., R. Nakamura, Y. Asano, and M. Imai. 1984. Distribution of alpha-adrenergic receptors in the rabbit nephron. Tohoku J. Exp. Med. 142:275-282.

10. Bello-Reuss, E. 1980. Effect of catecholamines on fluid reabsorption by the isolated proximal convoluted tubule. Am. J. Physiol. 238:F347-F352.

11. Imai, M. 1979. The connecting tubule: a functional subdivision of the rabbit distal nephron segments. Kidney Int. 15:346-356.

12. Iino, Y., J. L. Troy, and B. M. Brenner. 1981. Effects of catecholamines on electrolyte transport in cortical collecting tubule. $J$. Membr. Biol. 61:67-73.

13. Kimmel, P. L., and S. Goldfarb. 1984. Effects of isoproterenol on potassium secretion by the cortical collecting tubule. Am. J. Physiol. 246:F804-F810.

14. Stiles, G. L., M. G. Caron, and R. J. Lefkowitz. 1984. $\beta$ adrenergic receptors: biochemical mechanisms of physiological regulation. Physiol. Rev. 64:661-743.

15. Insel, P. A., and M. D. Snavely. 1981. Catecholamines and the kidney: receptors and renal function. Ann. Rev. Physiol. 43:625-636.

16. Imbert-Teboul, M., D. Chabardes, and F. Morel. 1980. Vasopressin and catecholamines sites of action along rabbit, mouse and rat nephron. Contrib. Nephrol. 21:41-47.

17. Morel, F. 1981. Sites of hormone action in the mammalian nephron. Am. J. Physiol. 240:F159-F164.

18. Chabardes, D., M. Imbert-Teboul, M. Montegut, A. Clique, and F. Morel. 1975. Catecholamine sensitive adenylate cyclase activity in different segments of the rabbit nephron. Pfluegers Arch. Eur. J. Physiol. 361:9-15.

19. Beck, N. P., S. W. Reed, H. V. Murdaugh, and B. B. Davis. 1972. Effects of catecholamines and their interaction with other hormones on cyclic $3^{\prime}, 5^{\prime}$-adenosine monophosphate of the kidney. $J$. Clin. Invest. 51:939-944.

20. Dousa, T. P., B. A. Jackson, and R. M. Edwards. 1980. Cellular action of vasopressin in medullary collecting tubules and in ascending limbs of Henle's loop. In Antidiuretic Hormones. S. Yoshida, L. Share, and K. Yagi, editors. Japan Scientific Societies Press, Tokyo; University Park Press, Baltimore. 193-211.

21. Kusano, E., J. L. Braun-Werness, D. J. Vick, M. J. Keller, and T. P. Dousa. 1983. Chlorpropamide action on renal concentrating mechanism in rats with hypothalamic diabetes insipidus. J. Clin. Invest. 72:1298-1313.

22. Jackson, B., R. M. Edwards, H. Valtin, and T. P. Dousa. 1980. Cellular action of vasopressin in medullary tubules of mice with hereditary mephrogenic diabetes insipidus. J. Clin. Invest. 66:110-122.

23. Kiebzak, G. K., A. N. K. Yusuf, E. Kusano, J. L. BraunWerness, and T. P. Dousa. 1985. ATP and cyclic AMP system in the in vitro response of the microdissected cortical tubules to PTH. Am. J. Physiol. 248:F152-F159.

24. Morel, F., D. Chabardes, and M. Imbert-Teboul. 1976. Methodology for enzymatic studies of isolated tubular segments: adenylate cyclase. In Methods in Pharmacology. Renal Pharmacology. M. Martinez-Maldonado, editor. Plenum Press, New York. 297-323.

25. Kriz, W., and B. Kaissling. 1978. Anatomical and ultrastructural studies upon heterogeneity of nephrons in the rabbit kidney. Proc. VIIth Int. Cong. Nephrol. S. Karger, New York. 217-223.

26. Bulger, R. E., R. E. Cronin, and D. C. Dobyan. 1979. Survey of the morphology of the dog kidney. Anat. Rec. 194:41-66.

27. Imai, M., and S. Sasaki. 1980. Physiological significance of the distribution of vasopressin dependent adenylate cyclase in the nephron. In Antidiuretic Hormone. S. Yoshida, L. Share, and K. Yagi, editors. 
Japan Scientific Societies Press, Tokyo; University Park Press, Baltimore. 175-191.

28. Morel, F. 1983. Regulation of kidney functions by hormones: a new approach. Rec. Prog. Horm. Res. 39:271-304.

29. Bernstein, K. N., and D. T. O'Connor. 1984. Antiadrenergic antihypertensive drugs: their effect on renal function. Annu. Rev. Pharmacol. Toxicol. 24:105-120.

30. Kawashima, H., S. Torikai, and K. Kurokawa. 1981. Localization of 25-hydroxyvitamin $D_{3} 1 \alpha$-hydroxylase and 24-hydroxylase along the rat nephron. Proc. Natl. Acad. Sci. USA. 78:1199-1203.

31. Maguire, M. E., E. M. Ross, and A. G. Gilman. 1977. $\beta$ adrenergic receptor: ligand binding properties and the interaction with adenylyl cyclase. In Advances in Cyclic Nucleotide Research, Vol. 8. P. Greengaard and G. A. Robinson, editors. Raven Press, New York. 2-74.

32. Bravo, E. L., R. C. Tarazi, R. W. Gifford, and B. H. Stewart. 1979. Circulating and urinary catecholamines in pheochromocytoma: diagnostic and pathophysiologic implications. New Engl. J. Med. 301: 682-686.
33. Nadeau, R. A., and J. de Champlain. 1979. Plasma catecholamines in acute myocardial infarction. Am. Heart J. 98:548-554.

34. O'Donnell, S. R., and J. C. Wanstall. 1981. Demonstration of both $\beta_{1}$ - and $\beta_{2}$-adrenoreceptors mediating relaxation of isolated ring preparations of rat pulmonary artery. Br. J. Pharmacol. 74:547-552.

35. Dousa, T. P. 1982. Localization of hormones and drugdependent cAMP systems in specific nephrol segments. In Advances in Pharmacology and Therapeutics II, Vol. 3, Cardio-Renal and Cell Pharmacology. H. Yoshida, Y. Hagihara, and S. Ebashi, editors. Pergamon Press, Oxford and New York. 295-300.

36. Ruggles, B. T., N. Murayama, J. L. Werness, S. Gapstur, M. D. Bentley, and T. P. Dousa. 1985. The vasopressin-sensitive adenylate cyclase in collecting tubules and in thick ascending limb of Henle's loop of human and canine kidney. J. Clin. Endocrinol. Metab. 60:914-921.

37. Murayama, N., J. L. Werness, E. Kusano, S. Christensen, and T. P. Dousa. 1984. Interaction of forskolin with vasopressin-sensitive cyclic AMP system in renal medullary tubules. J. Cyclic Nucleotide Protein Phosphoryl. Res. 9:427-433. 